Research Article

\title{
Phytochemical Screening and Potential Antibacterial Activity of Defatted and Nondefatted Methanolic Extracts of Xao Tam Phan (Paramignya trimera (Oliv.) Guillaum) Peels against Multidrug-Resistant Bacteria
}

\author{
Van-Anh Le Thi $\mathbb{D}^{\mathbb{1}},{ }^{1}$ Ngoc-Lien Nguyen $\mathbb{D}^{1},{ }^{1}$ Quang-Huy Nguyen $\mathbb{D}^{1,2}$ Quyen Van Dong, \\ Thi-Yen Do, ${ }^{1}$ and Kieu-Oanh Nguyen T. (D) ${ }^{1,2}$ \\ ${ }^{1}$ Department of Life Sciences, University of Science and Technology of Hanoi, Vietnam Academy of Science and Technology, \\ 18 Hoang Quoc Viet, Cau Giay, Hanoi, Vietnam \\ ${ }^{2}$ LMI-DRISA, University of Science and Technology of Hanoi, Vietnam Academy of Science and Technology, 18 Hoang Quoc Viet, \\ Cau Giay, Hanoi, Vietnam \\ ${ }^{3}$ Institute of Biotechnology, Vietnam Academy of Science and Technology, 18 Hoang Quoc Viet, Cau Giay, Hanoi, Vietnam
}

Correspondence should be addressed to Kieu-Oanh Nguyen T.; nguyen-thi-kieu.oanh@usth.edu.vn

Received 14 May 2021; Accepted 16 August 2021; Published 31 August 2021

Academic Editor: Carsten Wrenger

Copyright (C) 2021 Van-Anh Le Thi et al. This is an open access article distributed under the Creative Commons Attribution License, which permits unrestricted use, distribution, and reproduction in any medium, provided the original work is properly cited.

\begin{abstract}
Xao tam phan (Paramignya trimera (Oliv.) Guillaum) is a traditional herbal medicine in Vietnam. Previous investigations reported mainly compounds and bioactivities of roots, stems, and leaves while there is limited information about those of fruits. This study aims to reveal the difference in the chemical profile of defatted peel (DP) and nondefatted peel (NDP) methanolic extracts of $P$. trimera using colorimetric reactions and liquid chromatography coupled with high-resolution mass spectrometry (LC-HRMS) analysis. We also showed the potential antibacterial activity of two extracts against clinically isolated bacteria strains including P. aeruginosa, Salmonella sp., and $S$. aureus with the MIC values $<100 \mu \mathrm{g} / \mathrm{mL}$. This preliminary result proves the traditional usage of this herbal medicine and can be helpful for further investigation on the isolation and identification of the new compounds in P. trimera peels.
\end{abstract}

\section{Introduction}

The threat posed by antibiotic resistance became particularly critical in recent years because multiple and extended resistant bacteria, called "superbugs," have become more prevalent, leading to more common hard-to-treat infections all over the world $[1,2]$. This situation is a global problem but particularly pressing in developing countries where the infectious disease burden is high. Among these countries, Vietnam already experiences high antibiotic resistance levels, and an alarming increase of superbugs, resistant to powerful antibiotics, has been reported [3, 4]. Seriously, the COVID-19 situation has also aggravated antibiotic resistance. Statistics show that 50\% of COVID19 mortalities suffered from secondary infections caused by multidrug-resistant microorganisms (bacterial or fungal) or coinfections [5]. There is thus an urgent need to develop new antibacterial agents, especially against Gramnegative strains. While the investigation based on soil actinomycetes, the primary source of antibiotics, is useless because of the high rediscovery rate of known compounds [6], raising a novel candidate to conquer multidrug-resistant bacteria was thus crucial. Plant resource has long been used as herbal medicine to treat a vast array of inflammatory and bacterial infections [7]. Eventhough plants can produce a variety of secondary metabolites that are active to defend insects, microorganisms, herbivores, plant pathogens, and against human pathogens, the current study on the antimicrobial potential of plants is merely the tip of the iceberg. 
Paramignya trimera (Oliv.) Guillaum, locally named "Xao tam phan," belongs to the Paramignya genus of the Rutaceae family, mainly in the south of Vietnam [8]. This native plant is a traditional remedy used for liver protection and infectious disease treatment. Recently, various potential bioactivities, i.e., antioxidant, antibacterial, and anticancer activities and hepatoprotective property, were reported from the plant's roots, leaves, and stems. Many compounds that belong to coumarins, acridone alkaloids, phenols, flavonoids, and chromenes classes have been isolated from these tissues (Table 1S, Supplementary Materials) [9-19]. Furthermore, being a perennial woody plant, the exploitation of this plant from other tissues, i.e., fruits, instead of roots, leaves, or stems, may provide a chance to conserve this valuable medicinal plant. However, the research on the bioactivity and chemical composition of $P$. trimera fruits has been limited. This study thus aims to phytochemically dereplicate the defatted peel (DP) and nondefatted peel (NDP) methanolic extracts of $P$. trimera and to determine the activity against multidrug-resistant bacterial strains clinically isolated from patients in Vietnam.

\section{Materials and Methods}

2.1. Plant Materials. The Paramignya trimera fresh fruits were collected from the Ninh Hoa district, Khanh Hoa province, Vietnam, in August 2020. The samples were identified by Khanh Hoa Traditional Medicine Association, Khanh Hoa province, Vietnam. The voucher of specimens was deposited in the Department of Life Sciences, University of Science and Technology of Hanoi, Vietnam Academy of Science and Technology.

The whole fruits were cleaned in tap water, rinsed by distilled water to remove dust, and then separated into peel and seed. These tissues were ground into smaller pieces and stored at $-80^{\circ} \mathrm{C}$ until used for further analysis.

2.2. Microbial Materials. Bacterial strains including Acinetobacter baumannii, Pseudomonas aeruginosa, Escherichia coli, Staphylococcus aureus, and Salmonella sp. were kindly provided from LMI DRISA (Laboratoire Mixte International on Drug Resistant in South Asia, University of Science and Technology of Hanoi). These bacteria originally were isolated clinically from different hospitals in Hanoi, Vietnam, and maintained on the nutrient solution at a $-80^{\circ} \mathrm{C}$ deep freezer until experiments. The susceptibility test result of these strains against different antibiotics is provided in Table 2S (Supplementary Materials).

2.3. Sample Extraction. The fresh peels including the defatted peel (DP, defatted by $n$-hexane) and nondefatted peel (NDP) of $P$. trimera $(20 \mathrm{~g})$ were immersed in $100 \mathrm{~mL}$ of $\mathrm{MeOH}$ at room temperature for 24 hours. This process was repeated three times to extract the bioactive compounds maximally. The combined extracts were then filtered using the $0.45 \mu \mathrm{m}$ cellulose acetate membrane to remove all the particulars and concentrated in the Buchi Rotavapor (Flawil,
Switzerland) to obtain crude extracts. These extracts were dried under nitrogen gas blowing at room temperature to a constant weight.

2.4. Phytochemical Screening. Phytochemical screening of the abovementioned extracts was performed using the conventional protocol and reagents. Alkaloids were detected using Bouchardat/Mayer/Dragendorff tests. Indeed, to a small amount of each sample was added gently several drops of each reagent, Bouchardat, Mayer, and Dragendorff, in a test tube; the formation of brown/white/orange precipitate, respectively, confirms the presence of alkaloids. Flavonoids were identified by adding $2 \mathrm{~mL}$ of $10 \% \mathrm{NaOH}$ into the extracts; a yellow colour formation indicates the presence of flavonoids. This solution becomes colourless when adding few drops of $10 \% \mathrm{v} / \mathrm{v} \mathrm{HCl}$ solution. Glycosides were detected by mixing $5 \mathrm{~mL}$ of each extract and $25 \mathrm{~mL}$ of $10 \% \mathrm{v} / \mathrm{v} \mathrm{H}_{2} \mathrm{SO}_{4}$ solution. This mixture was heated to its boiling point for 15 minutes and then cooled and neutralized with $10 \% \mathrm{w} / \mathrm{v}$ $\mathrm{NaOH}$ solution. $5 \mathrm{~mL}$ of Fehling solution was added to it, and the red brick precipitate indicated the presence of glycosides. For terpenoids, $2 \mathrm{~mL}$ of dichloromethane was added to $5 \mathrm{~mL}$ of each extract, followed by carefully adding concentrated $\mathrm{H}_{2} \mathrm{SO}_{4}$. The formation of a reddish-brown colour layer confirms the presence of terpenoids. The $\mathrm{FeCl}_{3}$ test was used to identify tannins. A small amount of each extract is diluted and filtered, and then, few drops of $10 \% \mathrm{w} / \mathrm{v}$ solution of $\mathrm{FeCl}_{3}$ were added. The appearance of blue or green colour suggests the presence of tannins in the extract. For coumarins, $3 \mathrm{~mL}$ of $10 \% \mathrm{NaOH}$ was added to an aqueous plant extract, and yellow colour was observed in positive results. Saponins identified by shaking the mixture of $2 \mathrm{~mL}$ of alcohol diluted with water is added to $2 \mathrm{~mL}$ of the plant extract and shacked well for 15 minutes in a graduated cylinder. The formation of a layer of foam (approximately $1 \mathrm{~cm}$ ) indicates the presence of saponins. Steroids were detected by treating each extract with few drops of concentrated $\mathrm{H}_{2} \mathrm{SO}_{4}$ in dichloromethane; the appearance of red colour in the chloroform layer indicated the presence of steroids. Anthraquinones were detected by the Borntrager test, in which $1 \mathrm{~mL}$ of the ethyl-acetate fraction of each extract was added to $10 \mathrm{~mL}$ of $10 \% \mathrm{v} / \mathrm{v} \mathrm{NH}_{4} \mathrm{OH}$ solution. The formation of pink/red/violet colour indicates the presence of anthraquinones.

\subsection{Determination of Antibacterial Activity of the Extracts.} The inhibition percentage against bacterial strains was evaluated by the microbroth dilution assay by Sultanbawa et al. with some modifications [20]. First, the bacteria stock solution was taken out from the deep freezer and placed at room temperature. The $30 \mu \mathrm{L}$ bacterial supernatant was transferred into flash bottles containing $50 \mathrm{~mL}$ tryptic soy broth (TSB) and incubated under shaking condition $(150 \mathrm{rpm})$ at $37^{\circ} \mathrm{C}$ overnight. After $24 \mathrm{~h}$, the density of bacterial suspension was adjusted to equal the value of the $0.5 \mathrm{McF}$ arland standards corresponding to the final concentration of $10^{6} \mathrm{CFU} / \mathrm{mL}$ (colony-forming unit). 
The crude extracts (DP and NDP) were first dissolved in dimethyl sulfoxide (DMSO) at $20480 \mu \mathrm{g} / \mathrm{mL}$ and then diluted in the TSB medium at $1024 \mu \mathrm{g} / \mathrm{mL}$. Wells were filled with $100 \mu \mathrm{L}$ of each solution and $100 \mu \mathrm{L}$ bacterial suspension. Ciprofloxacin at a $30 \mu \mathrm{g} / \mathrm{mL}$ concentration was used as a positive control, while $2.5 \%$ DMSO was used as a negative control. Each plate well was normalized by the subtracted blank samples. Subsequently, the microplates were incubated at $37^{\circ} \mathrm{C}$ for 24 hours, and the samples' absorbance was measured at $600 \mathrm{~nm}$ using an iMark microplate reader (BioRad, California, USA). The inhibition percentage was calculated using the following formula: \%inhibition $=\left(A b \_D M S O-A b \_s a m p l e\right) / A b \_D M S O \times 100 \%$, where $\mathrm{Ab} \_\mathrm{DMSO}$ and Ab_sample are the absorbance values of the negative control and the sample, respectively.

The inhibition percentage of a series of dilutions ranging from 512 to $16 \mu \mathrm{g} / \mathrm{mL}$ was evaluated. The MIC value was defined as the concentration of extract exhibiting $70-80 \%$ bacterial inhibition after 24 hours of incubation at $37^{\circ} \mathrm{C}$. The MBC value was identified as the lowest concentration of extracts reducing the initial bacterial inoculum viability by more than $99.9 \%$ after 24 hours [21]. These values can be determined from the microbroth dilution of MIC tests. In plates containing the TSB medium, $10 \mu \mathrm{L}$ of culture taken from each well in the MIC range was inoculated. These plates were then incubated at $37^{\circ} \mathrm{C}$ for $24 \mathrm{~h}$. The lowest concentration of which each extract did not show any bacterial growth was regarded as their $\mathrm{MBC}$ values.

2.6. LC-HRMS Analysis. The extracts were dissolved in $\mathrm{MeOH}$ (HPLC grade) to $100 \mathrm{mg} / \mathrm{mL}$ solution, filtered by $0.22 \mu \mathrm{m}$ cellulose acetate membrane, and then transferred into the 2-mL vials for injection into the LC-HRMS system. The HPLC-DAD-ESI/QTOF-MS/MS analysis was performed on an ExionLC system coupled to an ExionLC DAD detector and equipped with a high-resolution X500 QTOF mass spectrometer (Sciex, USA). The SCIEX OS 1.0 software from Sciex (Sciex, USA) contains instrument control, data acquisition, data processing, and reporting functionality, all in one package. Chromatographic separation was achieved on a Kinetex XB-C18 $100 \AA$ column $(100 \mathrm{~mm} \times 2.1 \mathrm{~mm}, 1.7 \mu \mathrm{m})$ (Phenomenex). A binary mobile solvent was used: solvent $A\left(\mathrm{H}_{2} \mathrm{O}\right)$ and solvent $B$ $(\mathrm{MeOH})$. The mobile phase was pumped at a flow rate of $0.3 \mathrm{~mL} / \mathrm{min}$ with a gradient elution profile that began at $20 \% B$ at $5 \mathrm{~min}$, then linearly ramped to $80 \% B$ within $5 \mathrm{~min}$, ramped to $100 \% B$ in $5 \mathrm{~min}$, and held at $50 \% B$ for $5 \mathrm{~min}$; then, the column was reequilibrated at $25 \% B$ for $5 \mathrm{~min}$ before the next injection. The autosampler tray temperature was set to $15^{\circ} \mathrm{C}$, while the column temperature was $15^{\circ} \mathrm{C}$. The injection volume was $10 \mu \mathrm{L}$.

The signals were detected at a wavelength $\lambda 254 \mathrm{~nm}$. The QTOF HRMS was equipped with a TurbolonSpray ion source, and the ESI negative mode was applied, scanning spectra from $\mathrm{m} / \mathrm{z} 100$ to 3000 . The spray voltage and ion source temperature were set to $4500 \mathrm{~V}$ and $450^{\circ} \mathrm{C}$, respectively. The ion source gas 1 , ion source gas 2 , curtain gas, and CAD gas were set to 50,50, 25, and 7 psi, respectively. Metabolites annotation was based on UV, HRMS, MSMS spectra, and relative RTs.

2.7. Statistical Analysis. All experiments were performed in triplicate. The obtained data were analyzed using Microsoft Office Excel 2016 for statistical analysis. The independent Student's $t$-test and the one-way ANOVA test were used to assess the differences among different concentrations and between the test and negative control groups.

\section{Results and Discussion}

3.1. Phytochemical Screening. Preliminary phytochemical screening showed several groups of metabolites such as alkaloids, flavonoids, glycosides, terpenoids, coumarins, and steroids in the methanolic extracts of the nondefatted and defatted peels of $P$. trimera. The result of the phytochemical test is provided in Table 1.

Two extracts were rich in flavonoids and glycosides. These tests also showed relatively that alkaloids, flavonoids, and glycosides were found with higher concentrations in DP than NDP. Terpenoids and steroids, known as nonpolar compounds, dominate logically in nondefatted portion NDP rather than in DP. This phytochemical screening result was consistent with previous studies showing the profiles of roots and stems of $P$. trimera [9-19].

3.2. Antibacterial Activity of P. trimera Extracts. In 2017, the WHO published its first list of antibiotic-resistant "priority pathogens," a catalogue of 12 families of bacteria that pose the greatest threat to human health drawn up to guide and promote research and development of new antibiotics. According to the priority list of antibiotic-resistant bacteria, we focused on the five following most problematic bacteria in Vietnam: A. baumannii, P. aeruginosa, S. aureus, E. coli, and Salmonella sp. Indeed, eight clinical strains of the mentioned five species were used as models to test the antibacterial activity of the extracts. At the first screening step, the inhibition percentage of NDP and DP at the concentration of $512 \mathrm{mg} / \mathrm{mL}$ was nearly $100 \%$ against six multidrug-resistant strains, including $P$. aeruginosa PA1 and PA2, S. aureus SA1 and SA2, and Salmonella sp. SS1 and SS2 (Table 3S, Supplementary Materials). In particular, the DP represented a more substantial inhibitory capacity than NDP extracts, indicating that removing nonpolar compounds helps increase the activity.

These extracts were then subjected to determine the minimum inhibitory concentration (MIC) and minimum bactericidal concentration (MBC) values on six bacterial strains. Overall, all mentioned extracts can be considered bactericidal agents against six clinically dangerous bacteria in this study because their $\mathrm{MBC}$ values were no more than four times their MIC values. As given in Table 2, these extracts presented the MIC values globally ranging from 16 to $320 \mathrm{mg} / \mathrm{mL}$. The DP exhibited the highest antibacterial activity on all six strains with the MIC value $<80 \mathrm{mg} / \mathrm{mL}$, especially against four strains, i.e., $P$. aeruginosa $\mathrm{PA} 1, S$. aureus SA2, and Salmonella sp. SS 1 and SS2 with the MIC $<32 \mathrm{mg} / \mathrm{mL}$. At the 
TABLE 1: Phytochemical analysis of the nondefatted peel (NDP) and defatted peel (DP) of P. trimera.

\begin{tabular}{|c|c|c|c|}
\hline Test & Observation & NDP & $\mathrm{DP}$ \\
\hline \multicolumn{4}{|l|}{ Alkaloid } \\
\hline Bouchardat & Formation of brown precipitate & - & - \\
\hline Mayer & Formation of yellow-white precipitate & - & + \\
\hline Dragendorff & Formation of orange precipitate & - & + \\
\hline \multicolumn{4}{|l|}{ Flavonoids } \\
\hline Alkaline reagent & Appearance of yellow colour, become colourless when adding diluted $\mathrm{HCl}$ solution & ++ & +++ \\
\hline \multicolumn{4}{|c|}{ 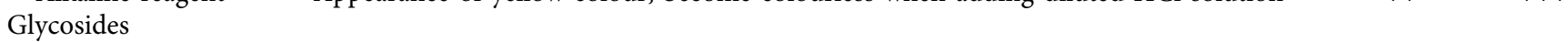 } \\
\hline Salkowski & Appearance of reddish colour & ++ & +++ \\
\hline Terpenoids & Formation of grey colour & ++ & + \\
\hline Tannins & Formation of blue or green colour & - & - \\
\hline Coumarins & Formation of yellow colour & ++ & ++ \\
\hline Saponins & Formation of foam & - & - \\
\hline Steroids & Appearance of red colour & ++ & + \\
\hline Anthraquinones & Appearance of pink/red/violet solution & - & - \\
\hline
\end{tabular}

TABLE 2: The minimum inhibitory concentration (MIC) $(\mu \mathrm{g} / \mathrm{mL})$ and the minimum bactericidal concentration $(\mathrm{MBC})(\mu \mathrm{g} / \mathrm{mL}) \mathrm{of}$ the nondefatted peel (NDP) and defatted peel (DP) methanolic extracts of $P$. trimera against six clinically isolated bacterial strains using the broth dilution assay.

\begin{tabular}{lcccccc}
\hline & \multirow{2}{*}{ Sample } & \multicolumn{2}{c}{ P. aeruginosa } & \multicolumn{2}{c}{ S. aureus } & \multicolumn{2}{c}{ Salmonella sp. } \\
& & PA2 & PA1 & SA1 & SA2 & SS2 \\
\hline \multirow{2}{*}{ MIC } & NDP & 320 & 24 & - & 32 & 32 \\
& DP & 64 & 16 & 80 & 16 & 32 \\
\multirow{2}{*}{ MBC } & NDP & 512 & 64 & - & 64 & 128 \\
& DP & 256 & 64 & 128 & 32 & 128 \\
\hline
\end{tabular}

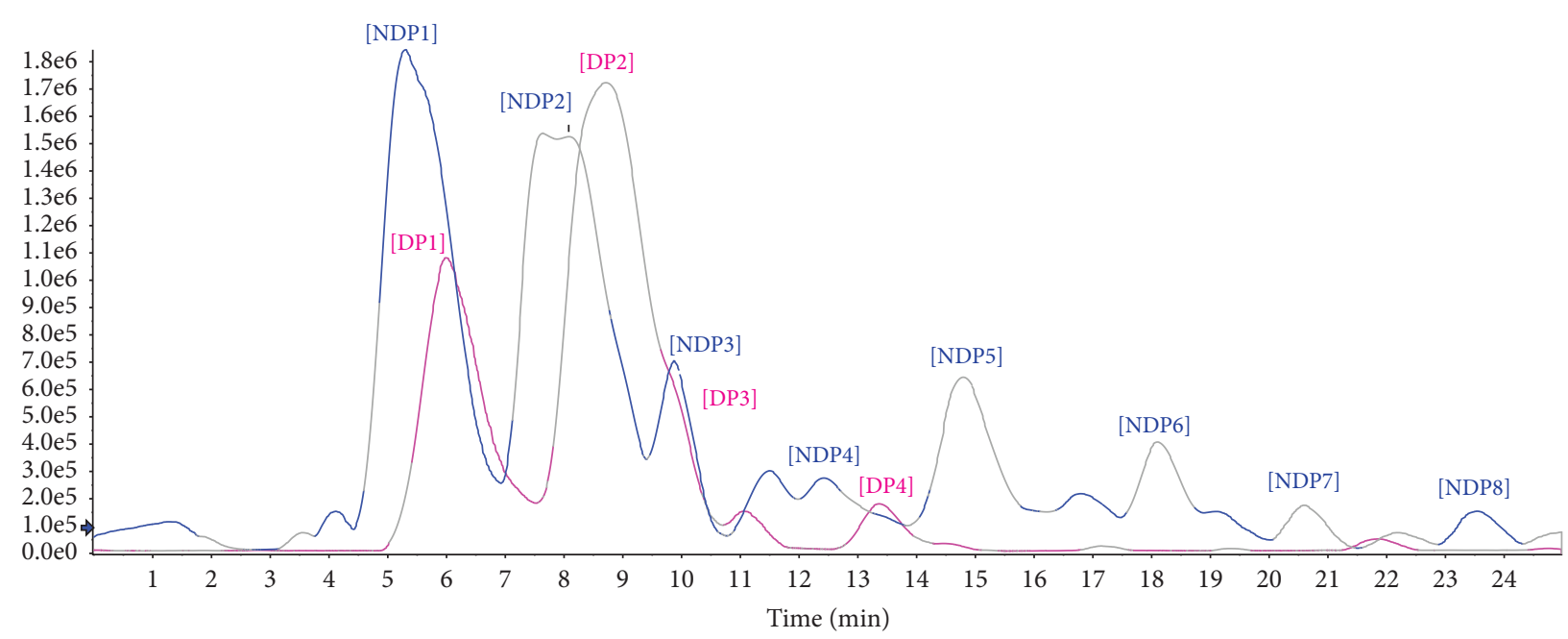

FIgURE 1: Total ion chromatograms of NDP (in blue) and DP samples (in pink) analyzed by HPLC-ESI-QTOF mass spectrometry.

same time, eventhough NDP did not show the potential activity on PA2 and SA1, this extract inhibited effectively four remaining strains with the $\mathrm{MIC}<32 \mathrm{mg} / \mathrm{mL}$. Indeed, the MIC variation followed a similar rule, horizontally and vertically, in Table 2, suggesting that the mechanism of action of these extracts seems to be the same on all six strains. The antibacterial activity was thus contributed by the same compounds pattern. Regarding the chemical profile of the extracts, we hypothesize that alkaloids may not be responsible for the bacterial inhibition activity, and this property can be due to flavonoids and coumarins.
$P$. aeruginosa is recognized as a common Gram-negative bacterium, an opportunistic pathogen associated with a range of nosocomial infections. In this study, the tested strains were isolated clinically from patients, with remarkable resistant mechanisms to many antibiotic classes (Table 2S, Supplementary Materials). Regarding the difference in the susceptibility tests, $P$. aeruginosa PA1 was resistant to all tested antibiotics except aztreonam. In contrast, PA2 was less resistant to quinolones (norfloxacin, ciprofloxacin, and levofloxacin). The inhibition activity of the extracts against PA2 was higher than against PA1 and 
TABLE 3: Metabolites annotation of the nondefatted peel (NDP) and defatted peel (DP) extracts of P. trimera.

\begin{tabular}{lccccc}
\hline Peak & Retention time & {$[\mathrm{M}-\mathrm{H}-](\mathrm{m} / \mathrm{z})$} & Formula & MS/MS fragmentation & Annotation \\
\hline NDP2; DP2 & $8.0 ; 8.6$ & 661.3898 & $\mathrm{C}_{43} \mathrm{H}_{52} \mathrm{O}_{3}$ & No fragmentation & Stearic acid \\
NDP3; DP3 & $10.1 ; 11.1$ & 283.2624 & $\mathrm{C}_{18} \mathrm{H}_{36} \mathrm{O}_{2}$ & 265.2512 & Dihydroxytetracosanoic acid \\
NDP4 & 12.3 & 399.3469 & $\mathrm{C}_{24} \mathrm{H}_{48} \mathrm{O}_{4}$ & $353.3418 ; 323.3310 ; 221.1022$ & Hydroxytetracosanoic acid \\
NDP5 & 14.8 & 383.3513 & $\mathrm{C}_{24} \mathrm{H}_{48} \mathrm{O}_{3}$ & 337.3459 & Tetracosanoic acid \\
NDP6 & 18.2 & 367.3567 & $\mathrm{C}_{24} \mathrm{H}_{48} \mathrm{O}_{2}$ & No fragmentation & Pentacosanoic acid \\
NDP7 & 20.7 & 381.3724 & $\mathrm{C}_{25} \mathrm{H}_{50} \mathrm{O}_{2}$ & $335.2577 ; 245.0265$ & Hexacosanoic acid \\
NDP8 & 23.5 & 395.3879 & $\mathrm{C}_{26} \mathrm{H}_{52} \mathrm{O}_{2}$ & No fragmentation & nd \\
DP4 & 13.4 & 758.5365 & nd & & \\
\hline
\end{tabular}

allowed us to hypothesize that the mechanism of action of these samples may be similar to that of quinolones. On the other hand, Salmonella sp. SS1 was sensitive to nalidixic acid, and SS2 was resistant to that antibiotic (Table 2S, Supplementary Materials). The MIC and MBC values of the extracts against these strains were relatively equal, suggesting that the activity may be controlled by different mechanisms with that of nalidixic acid. In the context that the effort to find out antibacterial agents against Gram-negative strains does not bring much hope in recent years, this result can be open to further more in-depth research on this studied plant tissue. For S. aureus, a Gram-positive species, the antibacterial activity of the extracts against SA2 was stronger than against SA1. The inhibition against $S$. aureus of the essential oil extracted from $P$. trimera leaves has been reported in the previous study [22]; however, it is the first study that indicates the potential antibacterial activity of peels of this plant. This is worth waiting because both SA1 and SA2 were dangerous multidrug-resistant bacteria (Table 2S, Supplementary Materials).

3.3. LC-HRMS Analysis. To go deeply into the phytochemical composition of these extracts above, we dereplicated known metabolites using LC-HRMS analysis. The exact mass of detected components was compared to those of reference compounds shown in previous studies [9-19] (Table 1S, Supplementary Materials). The QTOF MS-based chromatograms on nondefatted and defatted peels clearly showed similar and different segments between the two samples (Figure 1). Eventhough there is a slight shift in the peaks' retention time, two chromatograms demonstrated the same pattern in the first elution segment. The chromatogram of DP shows fewer peaks than that of the NDP sample due to the removal of hydrophobic compounds. Indeed, the peak eluted at $5.4 \mathrm{~min}$ in NDP and $5.8 \mathrm{~min}$ in the DP sample showed the base peak of molecular ion [M-H-] at $\mathrm{m} / \mathrm{z}$ 191.0555, corresponding to the formula $\mathrm{C}_{7} \mathrm{H}_{12} \mathrm{O}_{6}$. We annotated this peak as quinic acid based on the similarities in MS/MS fragmentation between the obtained peak and reference (Table 3). The peak NDP3 and DP3 showed the base peak at $\mathrm{m} / \mathrm{z} 283.2624$, which corresponds to $\mathrm{C}_{18} \mathrm{H}_{36} \mathrm{O}_{2}$. The peaks NDP6, NDP7, and NDP8 showed the base peaks at $\mathrm{m} / \mathrm{z}$ 367.3567, 381.3724, and 395.3879, respectively, corresponding to $\mathrm{C}_{24} \mathrm{H}_{48} \mathrm{O}_{2}, \mathrm{C}_{25} \mathrm{H}_{50} \mathrm{O}_{2}$, and $\mathrm{C}_{26} \mathrm{H}_{52} \mathrm{O}_{2}$. These formulas contain only two oxygens, one double bond, eluted at the end of chromatograms separated by reverse phase column and were detected only in the NDP samples suggesting that they were nonpolar metabolites. We annotated them as fatty acids: NDP3/DP3 (stearic acid), NDP6 (tetracosanoic acid), NDP7 (pentacosanoic acid), and NDP8 (hexacosanoic acid). The inhibitory effect of DP extract was more potent than NDP extract, suggesting that fatty acids do not contribute to the antibacterial activity. It was consistent with the hypothesis that antibacterial potential may be responsible by flavonoids and coumarins in $P$. trimera peel. Noticeably, the LC-HRMS analysis showed that detected peaks in both defatted and nondefatted peel extracts did not match to any of known metabolites previously isolated from other P. trimera tissues (Table 1S, Supplementary Materials). They have probably been never investigated in the peel of this medicinal plant; therefore, further studies should be performed to identify these compounds as well as its bioactivities.

\section{Conclusions}

For the first time, the potential inhibitory effect of the peels extracts of $P$. trimera against six MDR bacterial strains isolated clinically from hospitals in Vietnam, especially Gram-negative P. aeruginosa strains, was reported. Removal of the fatty portion of the peels could enhance the effectiveness against multidrug-resistant bacteria suggesting that the flavonoids and coumarins dominating in the DP may be responsible for the activity. This study contributes to the scientific validity of $P$. trimera being used traditionally as a medicine and provides the guide for further investigation of new compounds in $P$. trimera to develop new treatment options against multidrug-resistant bacteria.

\section{Data Availability}

The data used to support the findings of this study are included in the Supplementary Material file.

\section{Conflicts of Interest}

The authors declare that there are no conflicts of interest.

\section{Acknowledgments}

The authors acknowledge the Laboratoire Mixte International Drug Resistant in South Asia (LMI-DRISA) to provide the bacterial strains and Institute of Research for Development (IRD), France, to support the publication charge. 
The authors would also like to thank Institute of Chemistry, Vietnam Academy of Science and Technology, where the HPLC-DAD-ESI/QTOF system is located for facilitating our LC-HRMS analysis. This work was supported by University of Science and Technology of Hanoi through research grant USTH.BIO.01/18-19.

\section{Supplementary Materials}

The supplementary materials include 3 tables. (Supplementary Materials)

\section{References}

[1] M. Ahmad and A. U. Khan, "Global economic impact of antibiotic resistance: a review," Journal of Global Antimicrobial Resistance, vol. 19, pp. 313-316, 2009.

[2] A. C. Singer, H. Shaw, V. Rhodes, and A. Hart, "Review of antimicrobial resistance in the environment and its relevance to environmental regulators," Frontiers in Microbiology, vol. 7, p. 1728, 2016.

[3] S. B. Zaman, M. A. Hussain, R. Nye, V. Mehta, K. T. Mamun, and N. Hossain, "A review on antibiotic resistance: alarm bells are ringing," Cureus, vol. 9, no. 6, p. e1403, 2017.

[4] K. V. Nguyen, N. T. Thi Do, A. Chandna et al., "Antibiotic use and resistance in emerging economies: a situation analysis for Viet Nam,” BMC Public Health, vol. 13, no. 1, p. 1158, 2013.

[5] L. Rossato, F. J. Negrão, and S. Simionatto, "Could the COVID-19 pandemic aggravate antimicrobial resistance?" American Journal of Infection Control, vol. 48, no. 9, pp. 1129-1130, 2020.

[6] K. Lewis, "Riddle of biofilm resistance," Antimicrobial Agents and Chemotherapy, vol. 45, no. 4, pp. 999-1007, 2001.

[7] M. M. Cowan, "Plant products as antimicrobial agents," Clinical Microbiology Reviews, vol. 12, no. 4, pp. 564-582, 1999.

[8] B. T. T. Linh, D. H. Phu, and N. T. Nhan, "Investigation on chemical constituent of the chloroform extract of the stem of parapmignya trimera (Olivier) Burkill (Rutaceae)," in Proceeding of the Conference of Analytical Chemistry, Valencia, Spain, September 2015.

[9] M. T. T. Nguyen, P. H. Dang, T. N. Nguyen et al., "Paratrimerins $G$ and $H$, two prenylated phenolic compounds from the stems of Paramignya trimera," Phytochemistry Letters, vol. 23, pp. 78-82, 2018.

[10] N. T. Son, "Notes on the genus Paramignya: phytochemistry and biological activity," Bulletin of the Faculty of Pharmacy Cairo University, vol. 56, no. 1, pp. 1-10, 2018.

[11] P. H. Dang, T. H. Le, P. K. T. Phan, P. T. T. Le, M. T. T. Nguyen, and N. T. Nguyen, "Two acridones and two coumarins from the roots of Paramignya trimera," Tetrahedron Letters, vol. 58, no. 16, pp. 1553-1557, 2017.

[12] N. Trong Le, H. Duc Viet, T. Quoc Doan et al., "Biological activities of essential oils from leaves of Paramignya trimera (oliv.) Guillaum and limnocitrus littoralis (miq.) swingle," Antibiotics, vol. 9, no. 4, p. 207, 2020.

[13] V. T. Nguyen, M. C. Bowyer, Q. Van Vuong, I. A. V. Altena, and C. J. Scarlett, "Phytochemicals and antioxidant capacity of Xao tam phan (Paramignya trimera) root as affected by various solvents and extraction methods," Industrial Crops and Products, vol. 67, pp. 192-200, 2015.

[14] V. T. Nguyen, J. A. Sakoff, and C. J. Scarlett, "Physicochemical properties, antioxidant and anti-proliferative capacities of dried leaf and its extract from xao tam phan (Paramignya trimera)," Chemistry and Biodiversity, vol. 14, no. 6, Article ID e1600498, 2017.

[15] V. T. Nguyen, Q. V Vuong, M. C. Bowyer, I. A. Van Altena, and C. J. Scarlett, "Microwave-assisted extraction for saponins and antioxidant capacity from xao tam phan (Paramignya trimera) root," Journal of Food Processing and Preservation, vol. 41, no. 2, Article ID e12851, 2017.

[16] H. Le Tuan Anh, D.-C. Kim, W. Ko et al., "Anti-inflammatory coumarins from Paramignya trimera," Pharmaceutical Biology, vol. 55, no. 1, pp. 1195-1201, 2017.

[17] V. T. Nguyen, J. A. Sakoff, and C. J. Scarlett, "Physicochemical, antioxidant, and cytotoxic properties of xao tam phan (Paramignya trimera) root extract and its fractions," Chemistry and Biodiversity, vol. 14, no. 4, 2016.

[18] N. M. Cuong, T. T. Huong, P. N. Khanh et al., "Paratrimerins $A$ and $B$, two new dimeric monoterpene-linked coumarin glycosides from the roots and stems of Paramignya trimera," Chemical and Pharmaceutical Bulletin (Tokyo), vol. 63, no. 11, pp. 945-949, 2015.

[19] V. T. Nguyen, N. M. Q. Pham, Q. Van Vuong, M. C. Bowyer, I. A. van Altena, and C. J. Scarlett, "Phytochemical retention and antioxidant capacity of xao tam phan (Paramignya trimera) root as prepared by different drying methods," Drying Technology, vol. 34, no. 3, pp. 324-334, 2016.

[20] Y. Sultanbawa, A. Cusack, M. Currie, and C. Davis, "An innovative microplate assay to facilitate the detection of antimicrobial activity in plant extracts," Journal of Rapid Methods and Automation in Microbiology, vol. 17, no. 4, pp. 519-534, 2009.

[21] J. L. Ríos and M. C. Recio, "Medicinal plants and antimicrobial activity," Journal of Ethnopharmacology, vol. 100, no. 1, pp. 80-84, 2005.

[22] A. A. Aamer, M. Abdul-Hafeez, and S. Sayed, "Minimum inhibitory and bactericidal concentrations (MIC and MBC) of honey and bee propolis against multi-drug resistant (MDR) Staphylococcus sp. isolated from bovine clinical mastitis," Alternative \& Integrative Medicine, vol. 3, no. 4, pp. 1-9, 2014. 